\title{
Synchronization induced by periodic inputs in finite $N$-unit bistable Langevin models: The augmented moment method
}

\author{
Hideo Hasegawa 1 \\ Department of Physics, Tokyo Gakugei University, \\ Koganei, Tokyo 184-8501, Japan
}

(November 15, 2018)

\begin{abstract}
We have studied the synchronization induced by periodic inputs applied to the finite $N$-unit coupled bistable Langevin model which is subjected to cross-correlated additive and multiplicative noises. Effects on the synchronization of the system size $(N)$, the coupling strength and the cross-correlation between additive and multiplicative noise have been investigated with the use of the semi-analytical augmented moment method (AMM) which is the second-order moment approximation for local and global variables [H. Hasegawa, Phys. Rev. E 67 (2003) 041903]. A linear analysis of the stationary solution of AMM equations shows that the stability is improved (degraded) by positive (negative) couplings. Results of the nonlinear bistable Langevin model are compared to those of the linear Langevin model.
\end{abstract}

PACS No. 05.10.Gg, 05.45.-a, 05.45.Xt

Keywords bistable Langevin model, synchronization

\footnotetext{
${ }^{1}$ E-mail address: hideohasegawa@goo.jp
} 


\section{INTRODUCTION}

The Langevin model has been employed as a useful model for a wide range of stochastic phenomena in physics, biology and chemistry (for reviews, see [1, 2]). In the last decade, extensive studies have been made for the bistable Langevin model given by [3]-[20]

$$
\frac{d x_{i}}{d t}=x_{i}-x_{i}^{3}+\frac{J}{N} \sum_{j=1}^{N}\left(x_{j}-x_{i}\right)+I(t)+\xi_{i}(t)+x_{i} \eta_{i}(t), \quad i=1, \ldots, N,
$$

where $J$ denotes the diffusive coupling, $I(t)$ an applied input, and $\xi_{i}(t)$ and $x_{i} \eta_{i}(t)$ express additive and multiplicative noises, respectively [for detail, see Eqs. (5)-(7)]. Refs. [3]-[16] have studied the bistable Langevin model for a single element with $N=1$ and $J=0$ in Eq. (1). Because it is difficult to study the coupled bistable Langevin model given by Eq. (11) with an arbitrary $N$, previous studies adopted the mean-field model given by [17]-[20]

$$
\frac{d x}{d t}=(1-J) x-x^{3}+J \bar{x}+I(t)+\xi(t)+x \eta(x)
$$

which is valid for $N=\infty$. Here $\bar{x}=\langle x\rangle$ represents the time-dependent order parameter, and $\xi(t)$ and $x \eta(t)$ denote additive and multiplicative noises, respectively. The phase transition and synchronization in coupled bistable systems described by Eq. (2) have been investigated [17]-[20].

A common approach to finite- $N$ stochastic systems is to make direct simulations (DS) for the Langevin equation given by Eq. (11) or for relevant Fokker-Planck equation (FPE) [21. Despite a recent development in computers, it is not easy to perform DEs of Eq. (1) for appreciable values of $N$, which requires the computational time growing as $N^{2}$ with increasing $N$. For $N$-unit Langevin equations, the FPE method leads to $(N+$ 1)-dimensional partial equations to be solved with proper boundary conditions, which is usually very difficult. As a useful semi-analytical method for stochastic equations, Rodriguez and Tuckwell [22] proposed the moment method in which the first and second moments of variables are taken into account. In this approach, original $N$-dimensional Langevin equations are transformed to $(N / 2)(N+3)$-dimensional deterministic equations. This figure becomes 65 and 5150 for $N=10$ and $N=100$, respectively. Based on a macroscopic point of view, Hasegawa [23, 24] has proposed the augmented moment method (AMM), in which the dynamics of coupled Langevin equations is described by a small number (three) of quantities for averages and fluctuations of local and global variables. The AMM has been successfully applied to studies on the dynamics of coupled stochastic systems described by the linear Langevin model [24, 25], FitzHugh-Nagumo 
model [23, 26], Hodgkin-Huxley model [27] and networks [28]. The AMM is a semianalytical theory which is useful for a study of the finite- $N$ stochastic system, whereas conventional mean-field-type approximations are applied only to the $N=\infty$ case [17]-[20]. Cortical processing, for example, is performed by many coupled populations of neurons and each population consists of finite numbers of neurons. Effects of cluster sizes on the information processing in brain may be clarified in the AMM [29, 30].

Depending on the properties of elements forming a nonlinear system, we may classify them into two types A and B. In the type A, elements are excitable units or selfoscillators such as FitzHugh-Nagumo and Hodgkin-Huxley models. In the type B, units that form the system cannot oscillate on their own unlike excitable systems. An example of the type B is a bistable element, which has been used in biology, chemistry and for a study of neural networks [31]-33]. Interesting and intrigue properties of the bistable Langevin model have been extensively investigated [3]-[20]. Stationary probability distribution, first-passage time and the stochastic resonance for subthreshold periodic inputs in the bistable Langevin model subjected to cross-correlated additive and multiplicative white (or colored) noise have been studied. There are, however, still many unsolved basic problems. For example, dynamics of the finite- $N$ bistable Langevin model when time-dependent inputs are applied has not been well understood. The purpose of the present paper is to apply the AMM to the bistable Langevin model. We will investigate the dynamical response to external periodic inputs in the finite- $N$ bistable Langevin model subjected to additive and multiplicative noises with the use of the AMM. We apply suprathreshold inputs: deriving inputs are suprathreshold in the sense that they can induce the emergence of the transition of states from one of the bistable states to the other. We may investigate the synchronization of forced oscillations induced by suprathreshold periodical inputs in the type-B system, just as in the case of the type-A ensemble where the synchronization among self-oscillations are studied. We can examine effects on the synchronization of the size of systems, coupling strength and the cross-correlation between additive and multiplicative noises in a semi-analytical way within the AMM. The synchronization in the finite- $N$ coupled bistable model is recently investigated by numerical methods [34].

The paper is organized as follows. In Sec. 2, the AMM is applied to coupled bistable Langevin model subjected to additive and multiplicative noise with the cross-correlation. Numerical model calculations are presented in Sec. 3. In Sec. 4, we make a linear stability analysis of the stationary solution. The dynamical properties of the bistable Langevin 
model are compared to those of the linear Langevin model. Sec. 5 is devoted to our conclusion.

\section{Augmented moment method}

\subsection{Bistable Langevin model}

Generalizing the model given by Eq. (1), we adopt the $N$-unit coupled bistable Langevin model given by

$$
\frac{d x_{i}}{d t}=F\left(x_{i}\right)+\xi_{i}(t)+G\left(x_{i}\right) \eta_{i}(t)+I_{i}^{(c)}(t)+I^{(e)}(t)
$$

with

$$
I_{i}^{(c)}(t)=\frac{J}{Z} \sum_{k(\neq i)}\left[x_{k}(t)-x_{i}(t)\right] \quad(i=1, \ldots, N) .
$$

Here $F(x)=-\partial U(x) / \partial x, U(x)$ denotes the potential: $G(x)$ is an arbitrary function of $x$ : $J$ expresses the diffusive coupling: $Z$ (with $Z=N-1$ ) stands for the coordination number: $I^{(e)}(t)$ is an external input: $\xi_{i}(t)$ and $\eta_{i}(t)$ express zero-mean Gaussian white noises with correlations given by

$$
\begin{aligned}
\left\langle\eta_{i}(t) \eta_{j}\left(t^{\prime}\right)\right\rangle & =\alpha^{2} \delta_{i j} \delta\left(t-t^{\prime}\right), \\
\left\langle\xi_{i}(t) \xi_{j}\left(t^{\prime}\right)\right\rangle & =\beta^{2} \delta_{i j} \delta\left(t-t^{\prime}\right), \\
\left\langle\eta_{i}(t) \xi_{j}\left(t^{\prime}\right)\right\rangle & =\epsilon \alpha \beta \delta_{i j} \delta\left(t-t^{\prime}\right),
\end{aligned}
$$

where $\alpha$ and $\beta$ denote the strengths of multiplicative and additive noises, respectively, and $\epsilon$ the cross-correlation between additive and multiplicative noises.

We will study the dynamical properties of the coupled Langevin model with the use of the AMM [23, 24], in which the three quantities of $\mu, \gamma$ and $\rho$ are defined by

$$
\begin{aligned}
\mu(t) & =\langle X(t)\rangle=\frac{1}{N} \sum_{i}\left\langle x_{i}(t)\right\rangle, \\
\gamma(t) & =\frac{1}{N} \sum_{i}\left\langle\left[x_{i}(t)-\mu(t)\right]^{2}\right\rangle, \\
\rho(t) & =\left\langle[X(t)-\mu(t)]^{2}\right\rangle .
\end{aligned}
$$

Here $X(t)$ with $X(t)=N^{-1} \sum_{i} x_{i}(t)$ expresses a global variable, $\mu$ its mean, and $\gamma$ and $\rho$ denote fluctuations in local $\left(x_{i}\right)$ and global $(X)$ variables, respectively. Equations of 
motion for $\mu, \gamma$ and $\rho$ are given by (the argument $t$ being suppressed: for details, see appendix A)

$$
\begin{aligned}
& \frac{d \mu}{d t}=f_{0}+f_{2} \gamma+3 f_{4} \gamma^{2}+\frac{\phi}{2}\left\{\alpha^{2}\left[g_{0} g_{1}+3\left(g_{1} g_{2}+g_{0} g_{3}\right) \gamma\right]+\epsilon \alpha \beta\left(g_{1}+3 g_{3} \gamma\right)\right\}+I^{(e)} \\
& \frac{d \gamma}{d t}=2 f_{1} \gamma+6 f_{3} \gamma^{2}+(\phi+1)\left(g_{1}^{2}+2 g_{0} g_{2}\right) \alpha^{2} \gamma+2 \phi \epsilon \alpha \beta g_{2} \gamma+\left(\frac{2 J N}{Z}\right)(\rho-\gamma)+P \\
& \frac{d \rho}{d t}=2 f_{1} \rho+6 f_{3} \gamma \rho+(\phi+1)\left(g_{1}^{2}+2 g_{0} g_{2}\right) \alpha^{2} \rho+2 \phi \epsilon \alpha \beta g_{2} \rho+\frac{P}{N}
\end{aligned}
$$

with

$$
P=\alpha^{2} g_{0}^{2}+2 \epsilon \alpha \beta\left(g_{0}+g_{2} \gamma\right)+\beta^{2}
$$

where $f_{\ell}=(1 / \ell !) \partial^{\ell} F(\mu) / \partial x^{\ell}, g_{\ell}=(1 / \ell !) \partial^{\ell} G(\mu) / \partial x^{\ell}$, and $\phi=0$ and 1 in the Ito and Stratonovich representations, respectively. The $O\left(\gamma^{2}\right)$-order terms in Eqs. (11)-(13) are included with the use of the Gaussian approximation given by [23] [35]

$$
\begin{aligned}
\left\langle\left(\delta x_{i}\right)^{3}\right\rangle & \simeq 0 \\
\left\langle\left(\delta x_{i}\right)^{4}\right\rangle & \simeq 3\left\langle\left(\delta x_{i}\right)^{2}\right\rangle^{2} \\
\left\langle\left(\delta x_{i}\right)^{2}\left(\delta x_{j}\right)^{2}\right\rangle & \simeq 3\left\langle\left(\delta x_{i}\right)^{2}\right\rangle\left\langle\left(\delta x_{j}\right)^{2}\right\rangle .
\end{aligned}
$$

These terms play crucial roles for the bistable Langevin model although they are not necessary for the linear Langevin model [24]. Original $N$-dimensional stochastic equations given by Eqs. (3) and (44) are transformed to three-dimensional deterministic equations given by Eqs. (11)-(14).

For the bistable Langevin model with

$$
\begin{aligned}
& F(x)=x-x^{3}, \\
& G(x)=x,
\end{aligned}
$$

equations of motion are given by

$$
\begin{aligned}
& \frac{d \mu}{d t}=\mu-\mu^{3}-3 \mu \gamma+\frac{\alpha^{2} \mu}{2}+\frac{\epsilon \alpha \beta}{2}+I(t) \\
& \frac{d \gamma}{d t}=2\left(1-3 \mu^{2}-3 \gamma\right) \gamma+2 \alpha^{2} \gamma+\left(\frac{2 J N}{Z}\right)(\rho-\gamma)+P \\
& \frac{d \rho}{d t}=2\left(1-3 \mu^{2}-3 \gamma\right) \rho+2 \alpha^{2} \rho+\frac{P}{N}
\end{aligned}
$$


with

$$
P=\alpha^{2} \mu^{2}+2 \epsilon \alpha \beta \mu+\beta^{2}
$$

Eqs. (20) and (21) with $N=1(J=\epsilon=0)$ are in agreement with results obtained for a single bistable Langevin model [14], while those with $N=\infty(\rho=0)$ agree with results obtained for $N=\infty$ bistable Langevin model with a mean-field approximation [20]. For $J=0$, Eqs. (21) and (22) lead to

$$
\rho=\frac{\gamma}{N}
$$

which expresses the central-limit theorem.

A linear stability analysis of the stationary solution will be made in Sec. 4.1 with the use of the deterministic AMM equations given by Eqs. (20)-(22), as in the case of the coupled FitzHugh-Nagumo model [26].

\subsection{Synchrony}

In order to quantitatively study the emergence of a synchronized state of the ensembles defined by Eqs. (3) and (44), we first consider the quantity $S^{\prime}(t)$ given by

$$
S^{\prime}(t)=\frac{1}{N^{2}} \sum_{i j}<\left[x_{i}(t)-x_{j}(t)\right]^{2}>=2[\gamma(t)-\rho(t)] .
$$

When all variables are in the same state: $x_{i}(t)=X(t)$ for all $i$ (the completely synchronous state), we obtain $S^{\prime}(t)=0$ in Eq. (25). On the contrary, in the asynchronous state where $\rho=\gamma / N$, it is given by $S^{\prime}(t)=2(1-1 / N) \gamma(t) \equiv S_{0}^{\prime}(t)[23$. We may define the normalized ratio for the synchrony given by 23$]$

$$
S(t) \equiv 1-\frac{S^{\prime}(t)}{S_{0}^{\prime}(t)}=\left(\frac{N}{Z}\right)\left[\frac{\rho(t)}{\gamma(t)}-\frac{1}{N}\right],
$$

which is 0 and 1 for completely asynchronous $\left(S^{\prime}=S_{0}^{\prime}\right)$ and synchronous states $\left(S^{\prime}=0\right)$, respectively.

We may alternatively interpret $S(t)$ as the normalized mutual correlation given by

$$
S(t)=\frac{\zeta(t)}{\gamma(t)}
$$

with

$$
\begin{aligned}
\zeta(t) & =\frac{1}{N Z} \sum_{i} \sum_{j(\neq i)}\left[x_{i}(t)-\mu(t)\right]\left[x_{j}(t)-\mu(t)\right] \\
& =\left(\frac{N}{Z}\right)\left[\rho(t)-\frac{\gamma(t)}{N}\right] .
\end{aligned}
$$


We note that $S(t)=0$ for $J=0$ [Eq. (24)].

The equation of motion for $S(t)$ is given by

$$
\frac{d S}{d t}=-\left(\frac{P}{\gamma}\right) S+\frac{2 J}{Z}(Z S+1)(1-S),
$$

with the use of Eqs. (21), (22) and (26).

\section{Model Calculations}

We have performed model calculations, solving the AMM equations by the Runge-Kutta method with a time step of 0.01. Direct simulations for the $N$-unit Langevin model have been performed by using the Box-Mueller algorithm and the Euler method [36]-38] with a time step of 0.0001 . Results are averages of 1000 trials.

Our model given by Eqs. (3) and (4) includes five parameters of $N, J, \alpha, \beta$ and $\epsilon$. We will investigate effects of $N, J$ and $\epsilon$ for fixed values of $\alpha=0.1$ and $\beta=0.1$ otherwise noticed.

\section{Periodic pulse inputs}

We apply periodic pulse inputs at $t \geq t_{1}$ given by

$$
\begin{aligned}
I^{(e)}(t) & =A \sum_{k}\left[\Theta\left(t-t_{1}-k T_{p}\right) \Theta\left(t_{1}+k T_{p}+t_{w}-t\right)\right. \\
& \left.-\Theta\left(t-t_{1}-k T_{p} / 2\right) \Theta\left(t_{1}+k T_{p} / 2+t_{w}-t\right)\right]
\end{aligned}
$$

with $A=1.0, t_{1}=50, T_{p}=100$ and $t_{w}=10$ where $\Theta(x)$ denotes the Heaviside function: $\Theta(x)=1$ for $x \geq 0$ and zero otherwise. Figure 1(a), (b) and (c) show the time courses of $\mu(t), \gamma(t)$ and $S(t)$, respectively, for $N=10, J=0.2$ and $\epsilon=0.5$. Initial values of $x_{i}(t=0)$ are set to be -1.0 . When a positive pulse input $I(t)$ shown by the chain curve in Fig. 1(a) is applied at $t=50$ to the state which has been randomized by noise, the average value of $\mu$ is changed to about 1.0. When a negative pulse input is applied to the state with $\mu \simeq 1.0$ at $t=100$, the state is switched back to $\mu \simeq-1.0$. In these switching process, the local fluctuation $\gamma(t)$ and synchronization $S(t)$ are transiently increased. In

order to investigate the relation among $\mu, \gamma$ and $S, \gamma$ and $S$ calculated by the AMM are plotted as a function of $\mu$ in Fig. 1(d). It is shown that in the process of $\mu(t)$ changing from -1.0 to $+1.0, S(t)$ has the maximum value at $\mu \simeq 1.0$. In the reversed process, $S(t)$ has a maximum value at $\mu \simeq-1.0$. The maximum value of $S(t)$ for a process from 
$\mu \simeq-1.0$ to $\mu \simeq 1.0$ is smaller than that for the inversed process, which is due to the introduced cross-correlation $(\epsilon=0.5)$ between additive and multiplicative noise.

Similar $\mu-S$ plots for positive and negative correlations are depicted in Figs. 2(a) and (b), respectively. Figure 2(a) shows that with increasing $\epsilon$, the maximum value at $\mu \simeq 1.0$ $(\mu \simeq-1.0)$ is increased (decreased). The reversed behavior is realized for negative $\epsilon$, as shown in Fig. 2(b): the $\mu-S$ plot for negative $\epsilon$ is symmetric to that for positive $\epsilon$.

Figures 3(a) and (b) show the $J$ dependent $\mu-S$ plots for positive and negative $J$, respectively. From a comparison between Figs. 3(a) and (b), we note that the positive coupling is more effective than the negative one in increasing the synchrony: note that the vertical scale of Fig. 3(b) is smaller than that of Fig. 3(a).

The maximum value of $S, S_{\max }$, is plotted as a function of $N$ in Fig. 4 which shows that the synchrony is more increased for larger $J$ and smaller $N$.

\section{Sinusoidal inputs}

Next we apply sinusoidal inputs at $t \geq t_{1}$ given by

$$
I^{(e)}(t)=A \sin \left(\frac{2 \pi t}{T_{p}}\right) \Theta\left(t-t_{1}\right),
$$

with $A=1.0, t_{1}=50$ and $T_{p}=100$. Figures 5(a), (b) and (c) show time courses of $\mu$, $\gamma$ and $S$, respectively, and Fig. 5 (d) shows the relevant $\mu-S$ plot. From a comparison between Figs. 1 and 5, we note that the magnitudes of $\gamma$ and $S$ for the sinusoidal input are about three times larger than that for pulse input. The $\mu-S$ plots for periodic pulse and sinusoidal inputs are similar besides their magnitudes.

\section{Discussion}

\subsection{Stability analysis}

We will investigate the stability of the stationary solution of Eqs. (20)-(22), from which the Jacobian matrix is given by

$$
\left(\begin{array}{ccc}
1-3 \mu^{2}-3 \gamma+\frac{\alpha^{2}}{2} & -3 \mu & 0 \\
-12 \mu \gamma+2\left(\alpha^{2} \mu+\epsilon \alpha \beta\right) & 2\left(1-3 \mu^{2}-6 \gamma+\alpha^{2}\right)-\frac{2 J N}{Z} & \frac{2 J N}{Z} \\
-12 \mu \rho+\frac{2}{N}\left(\alpha^{2} \mu+\epsilon \alpha \beta\right) & -6 \rho & 2\left(1-3 \mu^{2}-3 \gamma+\alpha^{2}\right)
\end{array}\right) .
$$


In the case of $I=J=\epsilon=0$, we may analytically obtain stationary solutions and eigenvalues of the Jacobian matrix. Stationary solutions are divided into two cases $A$ and $B$ in which $\mu=0$ and $\mu \neq 0$, respectively: the latter is further classified to cases $B_{1}$ and $B_{2}$ as follows.

(1) Case $A$

Stationary solutions are given by

$$
\begin{aligned}
\mu^{2} & =0 \\
\gamma & =\frac{1}{6}\left(1+\alpha^{2}+\sqrt{D_{1}}\right), \\
\rho & =\frac{1}{6 N}\left(1+\alpha^{2}+\sqrt{D_{1}}\right),
\end{aligned}
$$

and relevant eigenvalues are given by

$$
\begin{aligned}
& \lambda_{1}=\frac{1}{2}\left[1-\sqrt{D_{1}}\right], \\
& \lambda_{2}=-2 \sqrt{D_{1}}, \\
& \lambda_{3}=1+\alpha^{2}-\sqrt{D_{1}},
\end{aligned}
$$

with

$$
D_{1}=\left(1+\alpha^{2}\right)^{2}+6 \beta^{2} .
$$

(2) Case $B_{1}$

Stationary solutions are given by

$$
\begin{aligned}
\mu^{2} & =\frac{1}{2}\left(1+\sqrt{D_{2}}\right), \\
\gamma & =\frac{1}{6}\left(1+\alpha^{2}-\sqrt{D_{2}}\right), \\
\rho & =\frac{1}{6 N}\left(1+\alpha^{2}-\sqrt{D_{2}}\right),
\end{aligned}
$$

and relevant eigenvalues are given by

$$
\begin{aligned}
\lambda_{1,2} & =-2-\sqrt{D_{2}} \pm \sqrt{4-3 D_{2}}, \\
\lambda_{3} & =-2+\alpha^{2}-2 \sqrt{D_{2}},
\end{aligned}
$$

with

$$
D_{2}=1-\alpha^{2}-\frac{\alpha^{4}}{2}-3 \beta^{2} .
$$


Table 1: Stationary solutions and eigenvalues within $O\left(\alpha^{2}\right)$ and $O\left(\beta^{2}\right)$

\begin{tabular}{|c||c|c|c|}
\hline & Case $A$ & Case $B_{1}$ & Case $B_{2}$ \\
\hline \hline$\mu^{2}$ & 0 & $\frac{1}{4}\left(4-\alpha^{2}-3 \beta^{2}\right)$ & $\frac{1}{4}\left(\alpha^{2}+3 \beta^{2}\right)$ \\
\hline$\gamma$ & $\frac{1}{6}\left(2+2 \alpha^{2}+3 \beta^{2}\right)$ & $\frac{1}{4}\left(\alpha^{2}+\beta^{2}\right)$ & $\frac{1}{12}\left(4+\alpha^{2}-3 \beta^{2}\right)$ \\
\hline$\rho$ & $\frac{1}{6 N}\left(2+2 \alpha^{2}+3 \beta^{2}\right)$ & $\frac{1}{4 N}\left(\alpha^{2}+\beta^{2}\right)$ & $\frac{1}{12 N}\left(4+\alpha^{2}-3 \beta^{2}\right)$ \\
\hline \hline$\lambda_{1}$ & $-\frac{1}{2}\left(\alpha^{2}+3 \beta^{2}\right)$ & $-2+2 \alpha^{2}+6 \beta^{2}$ & $\alpha^{2}+3 \beta^{2}$ \\
\hline$\lambda_{2}$ & $-2\left(1+\alpha^{2}+3 \beta^{2}\right)$ & $-4-\alpha^{2}-3 \beta^{2}$ & $-2-2 \alpha^{2}-6 \beta^{2}$ \\
\hline$\lambda_{3}$ & $-3 \beta^{2}$ & $-4+2 \alpha^{2}+3 \beta^{2}$ & $-3 \beta^{2}$ \\
\hline
\end{tabular}

(3) Case $B_{2}$

Stationary solutions are given by

$$
\begin{aligned}
\mu^{2} & =\frac{1}{2}\left(1-\sqrt{D_{2}}\right), \\
\gamma & =\frac{1}{6}\left(1+\alpha^{2}+\sqrt{D_{2}}\right), \\
\rho & =\frac{1}{6 N}\left(1+\alpha^{2}+\sqrt{D_{2}}\right),
\end{aligned}
$$

and relevant eigenvalues are given by

$$
\begin{aligned}
\lambda_{1,2} & =-2+\sqrt{D_{2}} \pm \sqrt{4-3 D_{2}}, \\
\lambda_{3} & =-2+\alpha^{2}+2 \sqrt{D_{2}} .
\end{aligned}
$$

When $\alpha$ and $\beta$ are small, stationary solutions and eigenvalues are expressed in powers of $\alpha$ and $\beta$ : those in the lowest approximation are summarized in Table 1 . We realize that the solution of $A$ is stable while that of $B_{2}$ is unstable because its first eigenvalue of $\lambda_{1}$ becomes positive. The solution of $B_{1}$ is stable if $\operatorname{Re}\left(\lambda_{1}\right)<0$ (see below).

It has been shown that although results of the stability condition derived by the second-order moment method are not in good agreement with those of numerical method, it yields semi-quantitatively meaningful results [20]. Bearing this fact in mind, we will study the stability condition for the case of $\mu \sim \pm 1$ (case $B_{1}$ ) with $I=\epsilon=0$ within the AMM by numerical methods. The solid curve in Fig. [6] shows the calculated boundary for $J=0.0$ within which the stationary solution is stable (results for $J \neq 0.0$ in Fig. [6 will be explained shortly). Figure 7(a) shows the $\alpha$ dependence of the maximum eigenvalues, $\lambda_{\max }$, for $\beta=0.0$ and $N=10$. For $J=0.0, \lambda_{\max }$ becomes zero for $\alpha=0.855$ ( $\beta=$ 
0.0), above which the stationary solution becomes unstable. When the coupling of $J$ is introduced, the critical $\alpha$ value becomes $0.738,0.968$ and 1.106 for $J=-0.2,0.2$ and 0.5 , respectively. Figure $7(\mathrm{~b})$ shows a similar plot of the $\beta$ dependence of $\lambda_{\max }$ for $\alpha=0.0$ and $N=10$. The solution becomes unstable for $\beta>0.577$ with $J=0.0, \alpha=0.0$ and $N=10$. With introducing $J$, the critical $\beta$ value is changed to $0.518,0.633$ and 0.712 for $J=-0.2,0.2$ and $J=0.5$, respectively. Similar calculations of $\lambda_{\max }$ are made for $\alpha=0.5$ with changing $J$. Squares, circles and triangles in Fig. 6 express results calculated for $J=0.5,0.2$ and -0.2 , respectively, which are shown by curves for a guide of eye. Figure 6] shows that the stability of the stationary solution against additive and multiplicative noise is improved (degraded) by positive (negative) couplings.

\subsection{Effect of the symmetry of $G(x)$}

It is necessary to point out that the symmetry in the $\mu-S$ plot depends on the symmetry of $G(x)$ for multiplicative noise. Indeed, in the case of $G(x)=x$ which has the odd symmetry: $G(x)=-G(-x)$, the asymmetry in the $\mu-S$ plot is obtained as shown in Figs. 2(a) and (b). However, if $G(x)$ has the even symmetry: $G(x)=G(-x)$, the asymmetry in the $\mu-S$ plot is not realized. We have performed the AMM calculation for $G(x)=x^{2}-1$ with the even symmetry, for which equations of motion for $\mu, \gamma$ and $\rho$ are given in appendix C. Calculated $S(t)$ and $\gamma(t)$ are plotted against $\mu(t)$ in Fig. 8(a) for $\epsilon \geq 0$ and in Fig. 8(b) for $\epsilon \leq 0$. With changing $\epsilon, \mu-S$ plot is little modified being symmetric independently of $\epsilon$, although magnitudes of the $\mu-\gamma$ plot is changed. A comparison of Figs. 8 (a) and (b) with Figs. 2(a) and (b) clearly shows that the symmetry of $G(x)$ is important in studying the effect of the cross-correlation between additive and multiplicative noise. This fact may be applied to effects of the cross-correlation on the stationary probability distribution as shown below. From the FPE in Eq. (A1), the stationary distribution $p(\boldsymbol{x})$ for $I=J=0$ in the Stratonovich representation $(\phi=1)$ is expressed by

$$
p(\boldsymbol{x})=\prod_{i=1}^{N} p\left(x_{i}\right)
$$

with

$$
\ln p(x) \sim \int \frac{2 F(x)}{\left[\alpha^{2} G(x)^{2}+2 \epsilon \alpha \beta G(x)+\beta^{2}\right]} d x-\left(\frac{1}{2}\right) \ln \left[\alpha^{2} G(x)^{2}+2 \epsilon \alpha \beta G(x)+\beta^{2}\right] .
$$


Straightforward calculations for $F(x)=x-x^{3}, G(x)=x$ and $G(x)=x^{2}-1$ lead to

$$
\begin{array}{rlrl}
p(x) \propto & \left(\alpha^{2} x^{2}+2 \epsilon \alpha \beta x+\beta^{2}\right)^{\left[\alpha^{2}+\beta^{2}\left(1-4 \epsilon^{2}\right)\right] / \alpha^{4}-1 / 2} & \\
& \times \exp \left[-\frac{\alpha^{2} x^{2}-4 \epsilon \alpha \beta x}{\alpha^{4}}-\frac{2 \epsilon\left(\alpha^{2}+\beta^{2}\left(3-4 \epsilon^{2}\right)\right)}{\alpha^{4} \sqrt{1-\epsilon^{2}}} \tan ^{-1}\left(\frac{\epsilon \beta+\alpha x}{\beta \sqrt{1-\epsilon^{2}}}\right)\right] \\
p(x) \propto & {\left[\alpha^{2}\left(x^{2}-1\right)^{2}+2 \epsilon \alpha \beta\left(x^{2}-1\right)+\beta^{2}\right]^{-\left(1 / 2 \alpha^{2}+1 / 2\right)}} & \text { for } G(x)=x \\
& \times \exp \left[\frac{\epsilon}{\alpha^{2} \sqrt{1-\epsilon^{2}}} \tan ^{-1}\left(\frac{\epsilon \beta+\alpha\left(x^{2}-1\right)}{\beta \sqrt{1-\epsilon^{2}}}\right)\right] & \text { for } G(x)=x^{2}-1 .
\end{array}
$$

Figures 9(a) and (b) show the stationary distributions for $G(x)=x$ and $G(x)=x^{2}-1$, respectively, with $\alpha=\beta=0.5$ for various $\epsilon$. For $G(x)=x$, an introduction of $\epsilon$ yields the asymmetry in $p(x)$, and $p(x)$ for a negative $\epsilon$ is anti-symmetric with that for a positive $\epsilon$ with respect to the $x=0$ axis. In contrast, for $G(x)=x^{2}-1, p(x)$ is symmetric independently of $\epsilon$, and an effect of a negative $\epsilon$ is different from that of a positive $\epsilon$. The difference in $G(x)$ reflects on various aspects of the bistable Langevin model such as the stationary distribution, the mean first-passage time and stochastic resonance, which have been conventionally calculated with the use of $G(x)=x[3]-[16]$.

\subsection{The linear Langevin model}

It is worthwhile to compare the properties of the nonlinear bistable Lanvevin model to those of the linear Langevin model. For the linear Langevin model with $F(x)=-\kappa x$ ( $\kappa$ : relaxation rate) and $G(x)=x$, we obtain equations of motion for $\mu, \gamma$ and $\rho$ in the Stratonovich representation as given by

$$
\begin{aligned}
& \frac{d \mu}{d t}=-\kappa \mu+\frac{\alpha^{2} \mu}{2}+\frac{\epsilon \alpha \beta}{2}+I(t), \\
& \frac{d \gamma}{d t}=-2 \kappa \gamma+2 \alpha^{2} \gamma+\left(\frac{2 J N}{Z}\right)(\rho-\gamma)+P, \\
& \frac{d \rho}{d t}=-2 \kappa \rho+2 \alpha^{2} \rho+\frac{P}{N},
\end{aligned}
$$

with

$$
P=\alpha^{2} \mu^{2}+2 \epsilon \alpha \beta \mu+\beta^{2}
$$

Equations (56)-(59) with $\epsilon=0$ agree with those previously obtained [24]. We may obtain analytic expressions for the stationary state, given by

$$
\mu=\frac{2 I-\epsilon \alpha \beta}{2 \kappa-\alpha^{2}}
$$




$$
\begin{aligned}
\gamma & =\frac{P}{2\left(\kappa-\alpha^{2}-J N / Z\right)}\left[1+\frac{J}{Z\left(\kappa-\alpha^{2}\right)}\right] \\
\rho & =\frac{P}{2 N\left(\kappa-\alpha^{2}\right)}
\end{aligned}
$$

yielding

$$
S=\frac{J}{J+Z\left(\lambda-\alpha^{2}\right)}
$$

where $P$ is given by Eq. (59) with $\mu$ in Eq. (60).

Figure 10 (a),(b) and (c) show time courses of $\mu, \gamma$ and $S$, respectively, for the linear Langevin model calculated with $\kappa=1.0$ and the same parameters for $N, J, \alpha, \beta$ and $\epsilon$ as in Fig. 1, The relevant $\mu-S$ plot is depicted in Fig. 10(d). A comparison of Fig. 10 with Fig. 1 shows that dynamical behavior of the bistable Langevin model is quite different from those of the linear counterpart. The nonlinearity in the bistable Langevin model plays an important role for the synchronization in the ensemble given by Eqs. (3) and (4).

We may make a linear analysis of the stationary solution given by Eqs. (60)-(62) . From Eqs. (56)-(58), we obtain the Jacobian matrix given by

$$
\left(\begin{array}{ccc}
-\kappa+\frac{\alpha^{2}}{2} & 0 & 0 \\
2 \alpha^{2} \mu & -2 \kappa+2 \alpha^{2}-\frac{2 J N}{Z} & \frac{2 J N}{Z} \\
\frac{2 \alpha^{2} \mu}{N} & 0 & -2 \kappa+2 \alpha^{2}
\end{array}\right)
$$

Eigenvalues are given by

$$
\begin{aligned}
& \lambda_{1}=-\kappa+\frac{\alpha^{2}}{2}, \\
& \lambda_{2}=-2 \kappa+2 \alpha^{2}-\frac{2 J N}{Z}, \\
& \lambda_{3}=-2 \kappa+2 \alpha^{2} .
\end{aligned}
$$

We note that eigenvalues in the linear Langevin model are independent of $I$ and $\beta$, which is different from those in the nonlinear bistable Langevin model.

\section{Conclusion}

We have studied the synchronization induced by periodic pulse and sinusoidal inputs in the $N$-unit bistable Langevin model subjected to cross-correlated additive and multiplicative noise with the use of the semi-analytical AMM [23, 24]. It has been shown that 
(1) the synchrony is transiently increased when the mean value of state variables is switched from one stable state to the other which is induced by an external suprathreshold input,

(2) the magnitude of synchrony is increased with increasing the coupling strength $(J)$ and/or decreasing the system size $(N)$,

(3) The stability of the stationary solution against additive and/or multiplicative noise is improved by positive couplings but degraded by negative couplings,

(4) the effect of the cross-correlation depends on its symmetry as well as a functional form of $G(x)$ for the multiplicative noise, and

(5) properties of the nonlinear bistable Langevin model are rather different from those of the linear Langevin model.

The AMM [23, 24] may be applied not only to the type-A stochastic ensembles of excitable elements but also to the type-B ones consisting of nonexcitable elements. It is expected possible to apply the AMM to various types of coupled stochastic systems. In the AMM we may easily solve the three-dimensional deterministic equations of $\mu, \gamma$ and $\rho$ for periodic as well as non-periodic (transient) inputs, although its applicability is limited to the small-noise case which is inherent in the moment method.

\section{Acknowledgements}

This work is partly supported by a Grant-in-Aid for Scientific Research from the Japanese Ministry of Education, Culture, Sports, Science and Technology.

\section{Appendix A: Derivation of the AMM equations}

The Fokker-Planck equation for the Langevin model given by Eqs. (3) and (44) is given by 24

$$
\begin{aligned}
\frac{\partial}{\partial t} p(\boldsymbol{x}, t) & =-\sum_{k} \frac{\partial}{\partial x_{i}}\left(\left[F\left(x_{i}\right)+I_{i}+\frac{\phi}{2}\left[\alpha^{2} G^{\prime}\left(x_{i}\right) G\left(x_{i}\right)+\epsilon \alpha \beta G^{\prime}\left(x_{i}\right)\right]\right] p(\boldsymbol{x}, t)\right) \\
& +\frac{1}{2} \sum_{i} \frac{\partial^{2}}{\partial x_{i}^{2}}\left(\left[\alpha^{2} G\left(x_{i}\right)^{2}+2 \epsilon \alpha \beta G\left(x_{i}\right)+\beta^{2}\right] p(\boldsymbol{x}, t)\right)
\end{aligned}
$$

where $p(\boldsymbol{x}, t)\left(\boldsymbol{x}=\left\{x_{k}\right\}\right), I_{k}=I_{k}^{(c)}+I, G^{\prime}(x)=d G(x) / d x$, and $\phi=1$ and 0 in the Stratonovich and Ito representations, respectively.

With the use of Eqs. (A1), equations of motion are given by [24]

$$
\frac{d\left\langle x_{i}\right\rangle}{d t}=\left\langle F\left(x_{i}\right)\right\rangle+\left\langle I_{i}\right\rangle+\frac{\phi \alpha^{2}}{2}\left\langle G^{\prime}\left(x_{i}\right) G\left(x_{i}\right)\right\rangle+\frac{\phi \epsilon \alpha \beta}{2}\left\langle G^{\prime}\left(x_{i}\right)\right\rangle,
$$




$$
\begin{aligned}
\frac{d\left\langle x_{i} x_{j}\right\rangle}{d t} & =\left\langle x_{i} F\left(x_{j}\right)\right\rangle+\left\langle x_{j} F\left(x_{i}\right)\right\rangle+\left\langle x_{i} I_{j}\right\rangle+\left\langle x_{j} I_{i}\right\rangle \\
& +\frac{\phi \alpha^{2}}{2}\left[\left\langle x_{i} G^{\prime}\left(x_{j}\right) G\left(x_{j}\right)\right\rangle+\left\langle x_{j} G^{\prime}\left(x_{i}\right) G\left(x_{i}\right)\right\rangle\right] \\
& +\frac{\phi \epsilon \alpha \beta}{2}\left[\left\langle x_{i} G^{\prime}\left(x_{j}\right)\right\rangle+\left\langle x_{j} G^{\prime}\left(x_{i}\right)\right\rangle\right] \\
& +\left[\alpha^{2}\left\langle G\left(x_{i}\right)^{2}\right\rangle+2 \epsilon \alpha \beta\left\langle G\left(x_{i}\right)\right\rangle+\beta^{2}\right] \delta_{i j}, \\
\frac{d\langle X\rangle}{d t} & =\frac{1}{N} \sum_{i} \frac{d\left\langle x_{i}\right\rangle}{d t}, \\
\frac{d\left\langle X^{2}\right\rangle}{d t} & =\frac{1}{N^{2}} \sum_{i} \sum_{j} \frac{d\left\langle x_{i} x_{j}\right\rangle}{d t},
\end{aligned}
$$

where $X=N^{-1} \sum_{i} x_{i}$. Expanding $x_{i}$ in Eqs. (A2)-(A5) around the average value of $\mu$ as

$$
x_{i}=\mu+\delta x_{i},
$$

we obtain equations of motion for $\mu, \gamma$ and $\rho$ given by Eq. (11)-(14) with the Gaussian approximation [Eqs. (15)-(17)] .

\section{Appendix B: The AMM equations for $G(x)=x^{2}-1$}

For the bistable Langevin model with

$$
\begin{aligned}
& F(x)=x-x^{3}, \\
& G(x)=x^{2}-1,
\end{aligned}
$$

Eqs. (11)-(14) yield equations of motion given by

$$
\begin{aligned}
& \frac{d \mu}{d t}=\mu-\mu^{3}-3 \mu \gamma+\alpha^{2} \mu\left(\mu^{2}-1+3 \gamma\right)+\epsilon \alpha \beta+I(t) \\
& \frac{d \gamma}{d t}=2\left(1-3 \mu^{2}-3 \gamma\right) \gamma+4 \alpha^{2} \gamma\left(3 \mu^{2}-1\right)+2 \epsilon \alpha \beta \gamma+\left(\frac{2 J N}{Z}\right)(\rho-\gamma)+P, \\
& \frac{d \rho}{d t}=2\left(1-3 \mu^{2}-3 \gamma\right) \rho+4 \alpha^{2} \rho\left(3 \mu^{2}-1\right)+2 \epsilon \alpha \beta \rho+\frac{P}{N}
\end{aligned}
$$

with

$$
P=\alpha^{2}\left(\mu^{2}-1\right)^{2}+2 \epsilon \alpha \beta\left(\mu^{2}-1+\gamma\right)+\beta^{2}
$$




\section{References}

[1] L. Gammaitoni, P. Hänggi, and F. Marchesoni, Rev. Mod. Phys. 70 (1998) 223.

[2] B. Lindner, J. García-Ojalvo, A. Neiman, and L. Schimansky-Gelíer, Physics Report 392 (2004) 321.

[3] D. J. Wu, L. Cao, and S. Z. Ke, Phys. Rev. E 50, 2496 (1994).

[4] C. Li, W. Da-jin and K. Sheng-zhi, Phys. Rev. E 52 (1995) 3228.

[5] Y. Jia and J. R. Li, Phys. Rev. E 53, 5786 (1996).

[6] Y. Jia and J. R. Li, Phys. Rev. Lett. 78, 994 (1997).

[7] Y. Jia and J. R. Li, Physica A 252, 417 (1998).

[8] L. Cao and D. J. Wu, Phys. Lett. A 260, 126 (1999).

[9] D. C. Mei, G. Z. Xie, and L. Cao et al., Phys. E 59, 3880 (1999).

[10] L. Cao and D. J. Wu, Phys. Rev. E 62, 7478 (2000).

[11] J. Wang, L. Cao and D. J. Wu, Phys. Lett. A 308 (2003) 23.

[12] C. W. Xie, D. C. Mei, and D. J. Wu, Eur. Phys. J B 33, 83 (2003).

[13] Y. Jin, W. Xu and M. Xu, Chaos, Solitons and Fractals 26 (2005) 1183.

[14] G-J. Zhang and J-X. Xu, Chaos, Solitons and Fractals 27 (2006) 1056.

[15] Z. Ping, Chinese J. Phys. 44, 117 (2006).

[16] H. Zhang, W. Xu and Y. Xu, Physica A 388 (2009) 781.

[17] R. C. Desai and R. Zwanzig, Statistical mechanics of a nonlinear stochastic model J. Stat. Phys. 19 (1978) 1.

[18] C. Van den Broeck, J. M. R. Parrondo, J. Armero, and A. Hern-'andez-Machado, Phys. Rev. E 49 (1994) 2639.

[19] M. Morillo, J. Gomez-Ordonez, and J. M. Casado. Phys. Rev. E 52 (1995) 316.

[20] Y-M. Kang and Y-L. Jiang, Chaos, Solitons and Fractals 41 (2009) 1987. 
[21] H. Risken: The Fokker-Planck Equation: Methods of Solution and Applications, Springer Series in Synergetics, Vol. 18 (Springer Verlag, Berlin, 1992)

[22] R. Rodriguez and H. C. Tuckwell, Phys. Rev. E 54 (1996) 5585.

[23] H. Hasegawa, Phys. Rev E 67 (2003) 041903.

[24] H. Hasegawa, J. Phys. Soc. Jpn. 75 (2006) 033001.

[25] H. Hasegawa, Physica A 374 (2007) 585.

[26] H. Hasegawa, Physica D 237 (2008) 137.

[27] H. Hasegawa, Phys. Rev E 68 (2003) 041909.

[28] H. Hasegawa, Phys. Rev E 70 (2004) 066107.

[29] H. Hasegawa, Phys. Rev. E 75 (2007) 051904.

[30] H. Hasegawa, Physica A 388 (2009) 499.

[31] H. Sompolinsky, Phys. Rev. A 34 (1986) 257.

[32] M. Camperi, X. Wang, J. Comput. Neurosci. 5 (1998) 383.

[33] A. Koulakov, S. Raghavachari, A. Kepecs, J. Lisman, Nature Neurosci. 5 (2002) 775.

[34] J. M. Casado, J. Gómez-Ordônez and M. Morillo, arXiv:0706.1541.

[35] S. Tanabe and K. Pakdaman, Phys. Rev. E 63 (2001) 31911.

[36] J. M. Sancho, M. S. Miguel, S. L. Katz, and J. D. Gunton, Phys. Rev. A 26 (1982) 1589.

[37] R. F. Fox, I. R. Gatland, R. Roy and G. Vemuri, Phys. Rev. A 38 (1988) 5938.

[38] D. C. Mei, Y. L. Xiang and C. W. Xie, Phys. Scr. 74 (2006) 123. 
Figure 1: (Color online) Time courses of (a) $\mu,(\mathrm{b}) \gamma$ and (c) $S$, and (d) $\gamma$ and $S$ as a function of $\mu$ for the pulse input with $N=10, J=0.2, \alpha=0.1, \beta=0.1$ and $\epsilon=0.5$. Solid and dashed curves an (a)-(c) denote results of AMM and DS, respectively: the chain curve in (a) shows input $I(t)$ shifted downward by - 2.0: arrows in (d) express the direction of time development.

Figure 2: (Color online) $\epsilon$ dependence of the $\mu-S$ plot: (a) for $\epsilon=0.0$ (the solid curve), $\epsilon=0.5$ (the dashed curve) and $\epsilon=0.9$ (the chain curve), and (b) for $\epsilon=0.0$ (the solid curve), $\epsilon=-0.5$ (the dashed curve) and $\epsilon=-0.9$ (the chain curve) with $N=10$ and $J=0.2$.

Figure 3: (Color online) $J$ dependence of the $\mu-S$ plot: (a) The $\mu-S$ plot for $J=0.1$ (the dashed curve), $J=0.2$ (the solid curve) and $J=0.5$ (the chain curve), and (b) for $J=-0.1$ (the dashed curve), $J=-0.2$ (the solid curve) and $J=-0.5$ (the chain curve) with $N=10$ and $\epsilon=0.0$.

Figure 4: (Color online) The maximum value of synchrony $S_{\max }$ against $N$ for $(J, \epsilon)=$ $(0.5,0.0)$ (the solid curve), $(0.2,0.0)$ (the dashed curve), and $(0.2,0.5)$ (the chain curve) with $\alpha=0.1$ and $\beta=0.1$.

Figure 5: (Color online) Time courses of (a) $\mu(t)$, (b) $\gamma(t)$ and (c) $S(t)$, and (d) $\gamma$ (the chain curve) and $S$ (the solid curve) as a function of $\mu$ for the sinusoidal input with $N=10, J=0.2, \alpha=0.1, \beta=0.1$ and $\epsilon=0.5$. Solid and dashed curves an (a)-(c) denote results of AMM and DS, respectively: the chain curve in (a) shows input $I(t)$ shifted downward by - 2.0: arrows in (d) express the direction of time development.

Figure 6: (Color online) The $\alpha-\beta$ phase boundary for $J=0.5$ (the chain curve), 0.2 (the dotted curve), 0.0 (the solid curve) and -0.2 (the dashed curve) calculated within the AMM with $N=10$ : the result for $J=0.0$ is calculated by $D_{2}=0$ [Eq. (46)], and marks (filled circles, squares and triangles) for $J \neq 0.0$ show numerical results with curves plotted for a guide of eye (see text). 
Figure 7: (Color online) (a) The maximum eigenvalue, $\lambda_{\max }$, as a function of $\alpha$ with $\beta=0.0$, and (b) $\lambda_{\max }$ as a function of $\beta$ with $\alpha=0.0$ for $J=-0.2$ (dashed curves), 0.0 (solid curves), 0.2 (dotted curves) and 0.5 (chain curves) with $N=10$ (see text).

Figure 8: (Color online) $S$ and $\gamma$ versus $\mu$ for pulse input with $G(x)=x^{2}-1$, (a) $S$ for $\epsilon=0.0$ (the solid curve) and $\epsilon=0.5$ (the dashed curve): $\gamma$ for $\epsilon=0.0$ (the chain curve) and $\epsilon=0.5$ (the dotted curve); (b) $S$ for $\epsilon=0.0$ (the solid curve) and $\epsilon=-0.5$ (the dashed curve): $\gamma$ for $\epsilon=0.0$ (the chain curve) and $\epsilon=-0.5$ (the dotted curve) $(N=10$ and $J=0.2$ ) (see text).

Figure 9: (Color online) The stationary distribution $p(x)$ for (a) $G(x)=x$ and (b) $G(x)=x^{2}-1$ for various values of $\epsilon$ with $\alpha=\beta=0.5$, the ordinate of (a) being different from that of (b).

Figure 10: (Color online) Time courses of (a) $\mu$, (b) $\gamma$ and (c) $S$, and (d) the $\mu-S$ plot for the pulse input applied to the linear Langevin model with $N=10, J=0.2, \lambda=1.0$, $\alpha=0.1, \beta=0.1$ and $\epsilon=0.5$. Solid and dashed curves an (a)-(c) denote results of AMM and DS, respectively: the chain curve in (a) shows input $I(t)$ shifted downward by -2.0 : arrows in (d) express the direction of time development. 
This figure "fig1.jpg" is available in "jpg" format from: http://arxiv.org/ps/0907.3955v3 
This figure "fig2.jpg" is available in "jpg" format from: http://arxiv.org/ps/0907.3955v3 
This figure "fig3.jpg" is available in "jpg" format from: http://arxiv.org/ps/0907.3955v3 
This figure "fig4.jpg" is available in "jpg" format from: http://arxiv.org/ps/0907.3955v3 
This figure "fig5.jpg" is available in "jpg" format from: http://arxiv.org/ps/0907.3955v3 
This figure "fig6.jpg" is available in "jpg" format from: http://arxiv.org/ps/0907.3955v3 
This figure "fig7.jpg" is available in "jpg" format from: http://arxiv.org/ps/0907.3955v3 
This figure "fig8.jpg" is available in "jpg" format from: http://arxiv.org/ps/0907.3955v3 
This figure "fig9.jpg" is available in "jpg" format from: http://arxiv.org/ps/0907.3955v3 
This figure "fig10.jpg" is available in "jpg" format from: http://arxiv.org/ps/0907.3955v3 